\title{
Design of a Dashboard of Software Metrics for Adaptable, Energy Efficient Applications
}

\author{
Vladimir Ivanov, Daria Larionova, Dragos Strugar, Giancarlo Succi \\ Innopolis University \\ Innopolis, Russia \\ \{v.ivanov, d.larionova, d.strugar, g.succi\}@innopolis.ru
}

\begin{abstract}
With the proliferation of internet-based software solutions worldwide came the need to maintain these products as well as to monitor the metrics collected within the products themselves. It has been a major challenge throughout the years to come up with an effective User Interface (UI) design that could be tailored to employees with multiple roles in the organization, especially in rapidly changing modern environments. Developing a self-adjusting dashboard not only could increase managers' productivity, but could potentially grow into a fully-fledged Adaptable System. However, such a system would carry a broad set of additional requirements. This paper presents the synergy of the adaptable systems in production and effective dashboard design according to the industry standards.
\end{abstract}

Index Terms-Adaptable Systems, Software Metrics, Energysaving Applications

\section{INTRODUCTION}

The goal of our ongoing research was to come up with a self-adjusting metric analysis system that would allow managers as well as engineers to make more informed decisions in the development of Adaptable, Energy Efficient solutions.

Developing a product first requires an understanding of the problem that is expected to be solved. Therefore, for this system a customizable dashboard can be used, which is a wellproven solution for this purpose.

Thus, we have created a web application to demonstrate our vision of the dashboard best-suited for this use case. The purpose of the global data collection system, to which the dashboard belongs to, is to present the obtained data in a specific structure and form, as well as to provide the users with an overview of the collected information.

Numerous academic papers emphasize the importance of the good design for the dashboard. In [4], Few points out that currently the entire purpose of dashboards is not only to display all the necessary information, but rather to provide a medium for communication and team collaboration.

The literature review section is primary based on the works of Zorin [7], [18], Pishulin [6], [12], Valiullin [16], whose preceding studies grounded the system that we am currently developing. It is also based on the work of Sarikaya, Correll, Bartram, Tory, and Fisher [13], who performed a systematic literature review on designing dashboards for different domains. These authors split the dashboards into types by different criteria, and explained the common patterns and features for each of them. Finally, it is based on the work of Yigitbasioglu, and Velcu [17], who also reviewed many sources for designing the dashboard, and suggested the mechanism for making decisions in their functionality.

Section II in greater detail the common problems which developers of the dashboard are likely to encounter. Section III summarizes fundamental goals and metrics of the GQM (Goal Question Metric) model, as required by software engineers for the evaluation of their performance. Section [V] covers possible features of the future dashboard. Section V lists some common visualization patterns. Section VI shows the overall design of the working solution. Then, sections VII and VIII talk about the Complex Adaptive Systems and how they map to our use case. And finally, section $[\mathrm{IX}$ gives the final thoughts and reflections.

\section{Problems Designation}

According to Valiullin [16], one of the main challenges is in selecting more appropriate metrics and displaying them in a meaningful and structured way. This choice has great influence on the understanding of those metrics. The dashboard should "provide intuitive, actionable, flexible, and programmable visualization to support effective decision making."

In addition, effective representation of selected metrics [16] is also a great challenge that we were facing. Solving this problem would allow users to easily detect and address the issues that may occur during the development process.

To solve these problems and choose more appropriate visualization techniques for more effective representation, Brath and Peters [1] suggest answering the following three questions:

- What metrics does the user need to see?

- What context does each metric require to make it meaningful?

- What is the visual representation that best communicates the metric?

In [14], the authors suggest to focus on the important metrics, with concise visualization. Such an approach that reduces redundancy by focusing on the goals obtained during the requirement collection \& analysis phase is very useful. Moreover, it helps to cope with making the wrong decisions in certain situations [17]. A similar view on data visualization is presented in [8], where the authors insist that the dashboard should be designed to be useful. This does not mean that it should contain all possible information that can be visualized, but only the necessary and sufficient data. The authors supplement this idea by suggesting the concepts of the "right" 


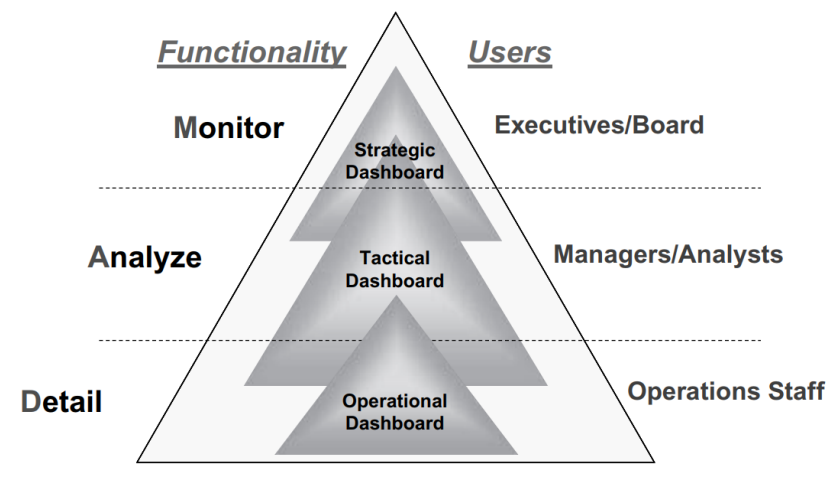

Fig. 1. Correspondence of the dashboard types and functionality. (Taken from [3. p. 103])

data and "right" visualization technique. Therefore, it is first necessary to determine which metrics should be displayed and explain the rationale of their choice. Second, choose the way to present them to the user, which can consequently minimize the time required for understanding them.

In addition, as noticed in [13], the design of the dashboard and its functionality greatly depends on its type. Few [4] and Eckerson [3] introduced three types of dashboards. Eckerson represents them in the form of a pyramid, with the operational dashboard type at the base, the tactical in the middle layer, and the strategic at the top (as shown in figure1). The three dashboard types differ in purpose and level of abstraction and interaction. According to this model, the strategic view is intended for monitoring the current situation. This allows minimum interaction with the user, contains an overview and consists of the most meaningful data. This is because, as mentioned in [13], the people often make screenshots and put them onto slides for showing a general picture. The purpose of the tactical view is to enable the analysis. This should contain more detailed information from the tactical view. The operational view should present a thorough form of the collected data and metrics. That would allow the user to summarize and also would help to find the reasons which led to this state, and come to possible solutions. In addition, according to the results of the surveys by Zorin in [18], the operational becomes more effective than the tactical and the strategic dashboard type.

In [17], Yigitbasioglu and Velcu suggest a path for making design decisions, that requires determining the four characteristics of a dashboard under development:

- its purpose is enabling

- consistency,

- monitoring,

- planning, and,

- communication,

- users and their

- tasks,

- knowledge and

- cognitive styles,
- design features (functional or visual) and

- outcomes, that is performance progress reached by this dashboard (i.e. improved speed, consistency etc.).

Taking into account that respondents of Zorin's surveys [18] are potential users, we can presume the variance in their education levels, age, company size and the position they are working on. Users prioritized their needs in the dashboard in the following order from the most to the least demanded:

1) performance monitoring;

2) planning;

3) communication;

4) measurement consistency.

Therefore these needs along with their priorities should be taken into account when architecting a new dashboard from scratch. The next couple of sections provide a more comprehensive description of how these can be formed in the GQM model.

\section{THE MAIN GOALS AND METRICS}

For selecting the necessary metrics in [8], the authors suggest using GQM+Strategies. This concept is a traditional Goal-Question-Metric approach supplemented by the links between different layers of organizational goals, i.e., highlevel and measurement goals. According to this approach, the authors ensure that a good dashboard should meet business goals. Hence, the development of such a dashboard requires the participation of all stakeholders.

As part of the study [18], Zorin conducted surveys with the representatives of software engineering companies, and extracted six common goals they wanted to achieve:

- Improving effort estimation efficacy;

- Using resources in a more efficient way;

- Executing testing activities in a more efficient and systematic way;

- Improving the quality of the development process;

- Completing projects successfully;

- Completing projects phases successfully.

Later in [7], Zorin summarizes them, and extracts three main goals:

- more effective effort estimation;

- more efficient use of resources;

- better software quality and development process.

The author divides the metrics needed for evaluating the achievement of these goals into five groups, displaying the:

- Progress status of the project;

- Speed of the work performed;

- Status of testing;

- Status of software quality;

- Effectiveness of effort estimation.

In addition, Zorin distinguishes the most frequent metrics:

1) Iteration Burndown chart;

2) Team velocity;

3) Code coverage; 
4) Effort estimation accuracy.

Finally, the author summarized the results in the GQM model, shown in figure 2

In [6], [12], Pishulin validated the results of the surveys by Zorin [7], [18]. The author investigated three goals highlighted in those studies, and determined the most suitable metrics for measuring them. For assessing the effectiveness of effort estimation, Pishulin identified the following key metrics:

1) Iteration Burndown;

2) Effort Estimation Accuracy;

3) Team Velocity.

For the evaluation of software quality and development process, the metrics are:

1) Passed Tests;

2) Code Coverage;

3) Unresolved Defects;

4) Class / Method Length;

5) Iteration Burndown;

6) Defect Removal;

7) Defected Density.

For the goal of more efficient use of resources, the author did not provide any information due to the lack of obtained information.

However, in [16], Valiullin suggested to spread this set of primary metrics and allow the user to create new metrics from existing ones by combining them in different ways. For describing the available manipulations, the author splits metrics to three types:

- raw,

- composite

- expression.

The raw metrics are those extracted directly from the collected user activities and source code (primary set of metrics described above). The composite metrics are the ones constructed from two raw or composite metrics by applying: 1. Simple arithmetic operations:

1.1. Addition;

1.2. Subtraction;

1.3. Multiplication;

1.4. Division;

2. Simple mathematical functions:

2.1. Average;

2.2. Maximum;

2.3. Minimum.

The expression metrics are obtained from one or more Raw or Composite type, and can be aggregated by some userdefined arithmetic expression.

\section{FUnCTIONALITY}

The main mission of the dashboard is allowing the user to monitor key metrics in terms of completion of predefined goals. Furthermore, the dashboard should provide various toolkits for tracking the development progress and product quality, and executing comparison of the current and perfect values [18]. Despite the amount of functionality, it is substantial to fit the dashboard to a single computer screen without compromising the content [4], [17].

In [8], the authors point out that understanding the displayed data should require the minimum effort from the user. The most relevant information should be provided with a "push" strategy. But at the same time, support interactivity with the ability to switch to "pull" mode. Moreover, the authors notice that the most relevant information should attract user's attention. However, at the same time, it is very important to find a balance and avoid a motley design. The authors suggest displaying the same data every time in the same place in order to make users become accustomed to the design.

One of the main features that the dashboard should implement is alerting users about the deviations of measurements from normal values. In [16], the author suggested notifying the user about abnormalities by coloring metric tiles according to their trend. For example, red could be used for going out of predefined by the user predefined range, green for those in the range and any other for neutral or default. [8], [12], [13] covered an idea of using arrows for showing the current tendency. The method in [3] can be useful for color-blind people to help them with correct interpretation of results. At the same time, it is significant not to overuse colors, visual structures and other catchy elements of design, in order not to overload the view [17].

According to [13], it is significantly important to make the dashboard customizable and adaptable to different users and situations. This objective can be obtained by allowing the users to set the goals, by themselves select the metrics to be displayed and set their own admissible and critical borders. Furthermore, the author draws our attention to the fact that filters [13], [16] and comparison support [13] are able to supplement the dashboard with more flexibility.

In [16], Valiullin continues this idea, and notes that the system under study should be adaptable for any project goal and provide multivariate analysis. The author states, that this adaptability can be satisfied with the implementation of roles and settings for choosing metrics for each project and goal individually. Furthermore, since users can work on several projects at the same time, for convenience of monitoring their status, the dashboard should provide support for multiple projects, and define separate sets of metrics and goals for all of them [16].

Another frequently mentioned concept lies in presenting different views on collected data and metrics, which can be reached by two ways.

The first one is to introduce roles with specific goals. Therefore, according to the goals, different analysis and visualization techniques should be selected with different points of view about the data [2]. In addition, roles can help to solve the problem of data visibility and privacy [13].

The second one is providing different levels of abstractions and different degree of details presented to the user. For introducing the general view about the situation, there should be a way to combine many metrics to several numbers, and 


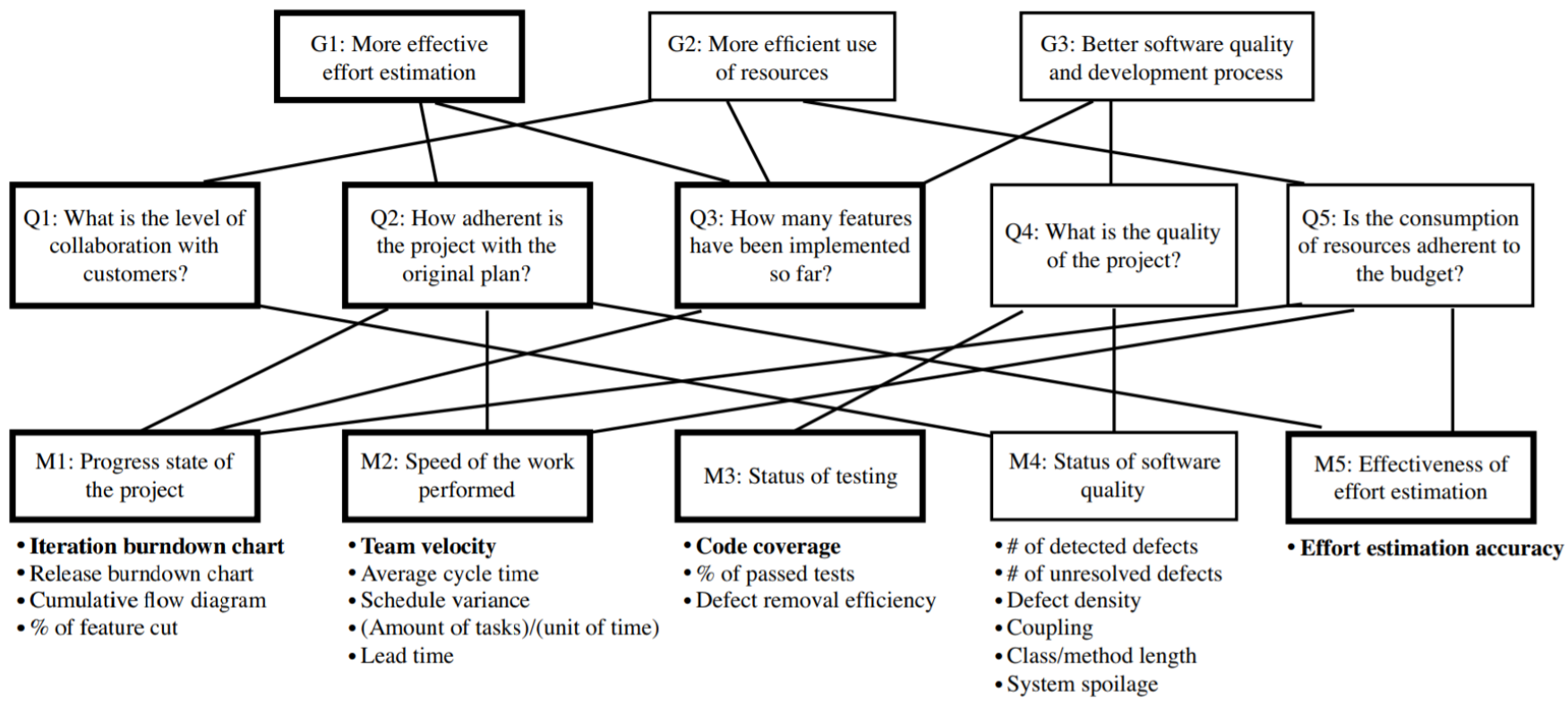

Fig. 2. Summary of the GQM with the most common aspects in bold (Taken from [7])

avoiding both detailed and redundant information [2]. More complete and thorough views can be reached by drill-down navigation or an "exploration mode" [4], [11], [13], [16]. It means that for "exploring" the nature of a certain result, it should be possible to switch between a generic and a more detailed view of the same metrics down to the raw data view. Using this technique would provide users with convenience in accuracy of data analysis, performed automatically [13].

According to [6], respondents want to have the ability to watch individual team members' metrics that can be satisfied by introducing hierarchical views to collected data,on the part of individual developers, teams and even the whole company [16]. Furthermore, interviewees would like the dashboard to display possible reasons of metrics changes and recommendations for recovering and improvement of current situation. Thus, the what-if simulation can take place [13], [18].

\section{Visualization}

Considering the structure of the dashboard in [16], Valiullin, relying on solutions from [8], [9] suggests the design based on Andon board with tiles, where each tile shows a numeric metric. This representation can speed up the understanding of the data and decision making as well as providing a unified way of metrics visualization in order to maintain the system scalability. However, there pointed a need in specific views for some metrics.

In [17], the authors make a investigation on data representation formats. Some researchers prefer graphical forms to tabular ones, whereas some place tables over graphs. Others do not exalt any of them believing that the choice of representation format highly depends on the task it is intended to solve. Graphs are more useful for tasks implicating comparisons and studying relationships of data, while the tabular form is more suitable for obtaining the certain information. Thereby, the authors admit possibility of switching to the displaying format more preferable by the user. In [16] for detailed view of the metrics, it is suggested to use two representations: values the metric consists of and the chart with its behaviour.

[5] suggests using different treemaps, texture, and bump mapping; animated zooming and panning for visualization of metrics. However, in [3] the author underlines that sometimes even at first glance simple features can bring additional complexity. The ease of use becomes one of the most important characteristic of any product. As noticed in [13], some authors even suggest reducing the interactivity for simplifying the system. Therefore, finding a balance in flexibility and customization is crucial.

\section{RESUlts}

This section focuses on establishing a strong relationship between the metrics and the goal. We are also going to present screenshots and various UI components we created. Results are presented in a form of a web page built with state-of-the-art front end web development library React.js.

As we noted earlier, the combination of flexibility and customization is a major factor in the User Experience (UX). That is precisely why we chose to create numerous widgets that users have control over. These widgets encapsulate graphs, charts, percentages, numerical values and time management tools alongside other key functionalities. They are designed to be easily reusable and re-sizeable. This also contributes to the fact that the solution is fully responsive and works on all screen sizes. Some of the examples you can see on figures 4 5. and 6 


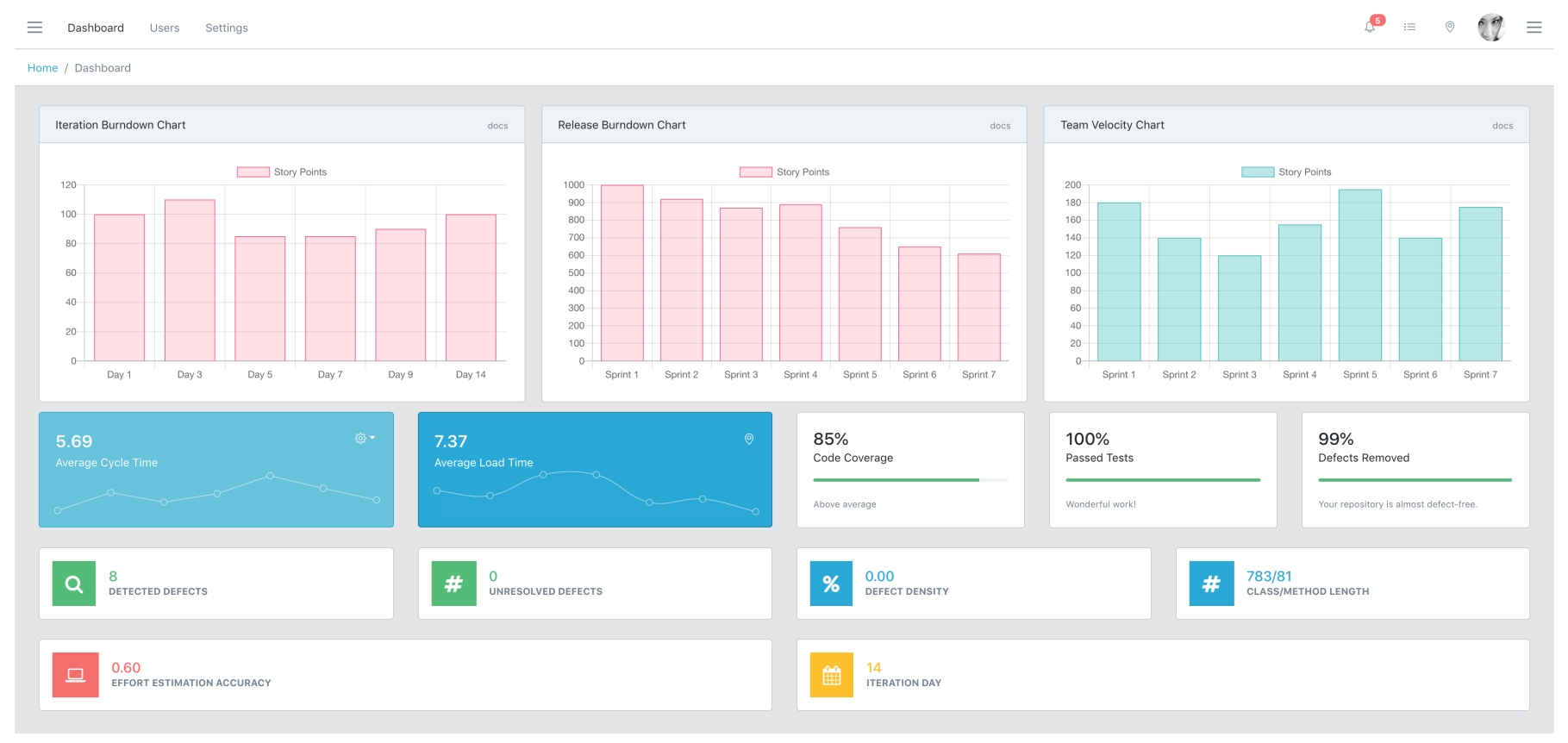

Fig. 3. HTML Web Interface Results (InnoMetrics)

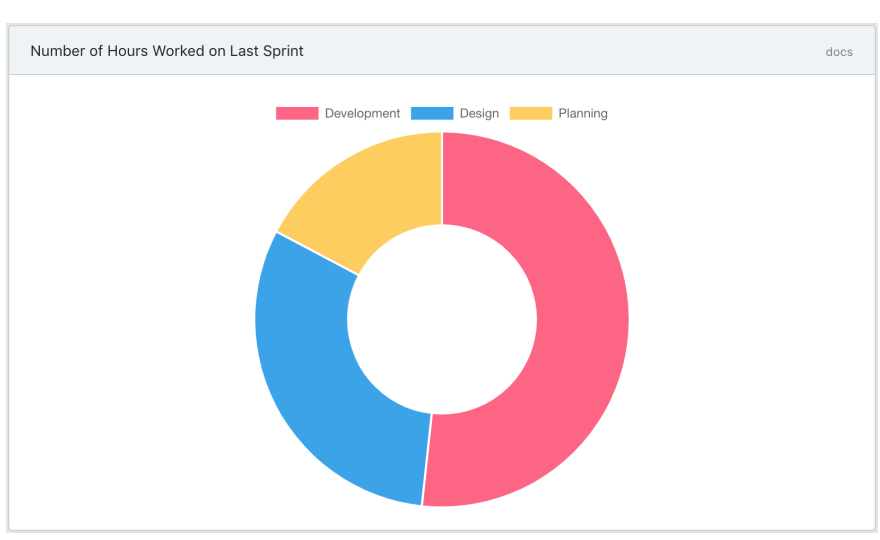

Fig. 4. Doughnut Graph Representation

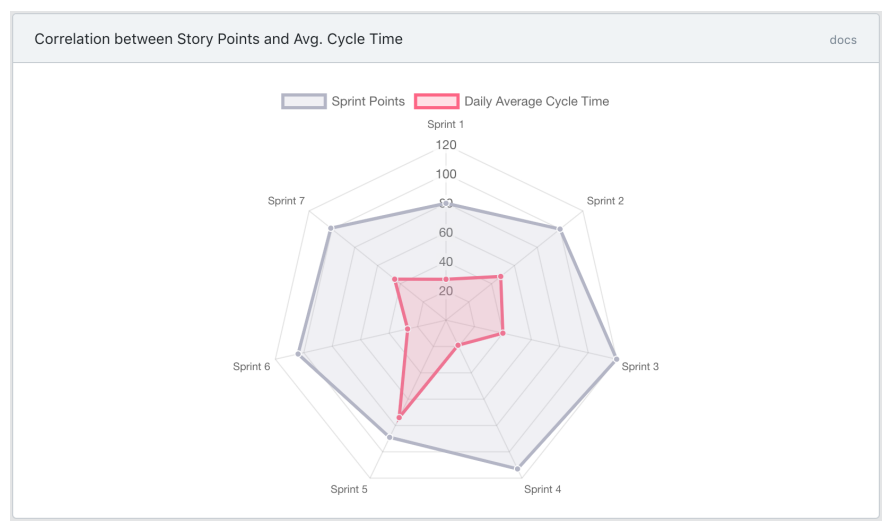

Fig. 5. Polar Graph Representation

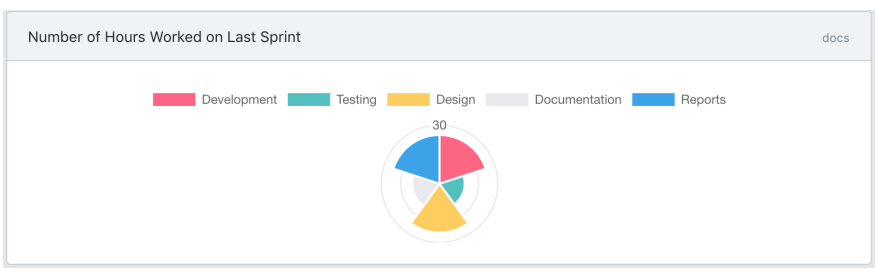

Fig. 6. Radial Graph Representation

Figure 3 showcases all the parts of the dashboard. The page contains 14 metrics selected for the study. Each of these metrics is placed in one of the widgets, or so called tiles. There is a total of 3 bar graphs, 2 line charts, 4 percentagebased values, 4 numerical values and 1 date value.

This prototype assumes that the software development team is using the Scrum methodology, with 7 iterations completed. Each iteration is two weeks long. The task of this prototype is to find out if the relationship between the metrics and the goal is obvious to the engineers. In addition, it is also very important to immediately see the most representative metrics for the Metric-Goal relationship.

This is precisely why we chose to make the connection with Adaptable systems. Not only would applying the notions from Complexity Theory allow the dashboard to present the most important metrics for certain situations, but it would also prevent it from being susceptible to change. 


\section{Complex AdAPtive Systems}

Realizing that the dashboard needs to adapt to rapid change in today's software development life-cycle, we suggest that modern dashboards should be a part of Complex Systems, more specifically, the Complex Adaptive Systems.

We represent each of the software development metrics, depicted as widgets on the 3, as agents within the system. Some of them are dependent on one another and some of them are not. The ones who are, are not strictly interacting in a linear, predictable way.

By embracing adaptation these agents have the potential to synchronize their internal states with the other agents in the system. Additionally, the system should be able to recognize these changes and self-adjust with the emergence of globally coherent patterns of adjustment developing.

Then, this Complex Adaptive System, the dashboard, should be able to feed back this information to micro-level agents. In order to preserve the relevance of certain metrics, the system makes the natural selection based on their fitness criteria to the environment. In our case, the fitness function is broadly defined as follows: "An agent has a higher contribution/fitness to the overall system if and only if a slight change in that specific metric would yield a significant change in the overall system fitness, the difference between the expected value and the actual value is above average, or a metric answers some custom questions that dashboard users may have". Applied and contextualized to our use case, the metrics are more relevant if the current value greatly differs from the expected one, or a slight change in a specific metric may yield a substantial increase in the well-being of the entire system.

Such a system embraces the complex dynamic between the micro-level components (metrics) and the overall system (the dashboard). The interaction between the differentiation of micro- and macro-level agents with different goals and agendas creates the core dynamic of complexity in our system.

Intrinsic to the Adaptive Systems is the notion of innovation; i.e. coming up with novel outcomes that we could not have predicted ahead of time. This is the crucial aspect of designing and developing a dashboard engineered to dynamically present the most useful metrics to the person using the dashboard. For example, a correlation between some two metrics may yield a significant improvement in the overall system functioning. However, that relationship has not been made beforehand. It is only by feeding the data to the system that it is able to come up with the correlations which may drastically change the overall system fitness.

The next two notions that need to explored are Non-linear Dynamics [15] and Dynamic Equilibrium [10].

\section{A. Non-linear Dynamics}

We realize that common assumptions that managers and stakeholders may have when creating and maintaining the dashboards for their products are very often not close to the real-world outcome. For example, they may think that one metric may be very important, and that turns out not to be true. And over time that results in a waste of their time looking at the metric and trying to improve it, while not focusing on the metrics that are actually significant at the moment. Put differently, the common assumptions that stakeholders may have about predicting the outcome based only on the initial input do not often work out in Complex Adaptive Systems due to their emergent complexity. Non-linear dynamics in adaptive systems is necessary to constantly change the internal states of the agents within the system, resulting in the change the entire system's state.

\section{B. Dynamic Equilibrium}

Utilizing the concept of the Dynamic Equilibrium allowed us to embrace our GQM model to come up with the state which has the following characteristics:

- the current state is never completely stable, which results in the full stagnation

- the current state is never in complete chaos, where there is nothing to bind individual actors together

- the current state is always in a so called "Dynamic Equilibrium" where all actors are loosely bound to each other with the plethora of room to innovate and improve

\section{APPlying THE COMPLEX SyStem TECHNIQUES TO OUR USE CASE}

As previously noted, the agents in the Complex System are represented as widgets. Each of these widgets encapsulates one metric that is used for maintaining and monitoring the software development process. Different visual representation apply to each of these metrics, of course. Some of them are more useful and easier to think about when depicted as continuous graphs, some of them as plan numbers, as seen on figure 3

It is then challenging to decide how these metrics can bring the variety, and the non-linear dynamics that Complex Systems impose. There is a total of 14 metrics. It is only by combining them that we will be able to achieve what we have suggested above. We needed to have a synergy between multiple metrics to see how they correlate to one another and how the increase in a specific factor or a group of factors can influence the whole system.

That is specifically why we decided to create custom widgets that represent the correlation between two or more metrics in the system. Even though such functionally complex widgets may contain a lot of data, as they are a relevant component to look after, they play a crucial role in the self-sustainability and continuous evolution of the dashboard system.

An example of such a combination is shown in the figure 7. One can immediately notice a very significant property. It is that the graph is now a complex structure consisting of two sub-graphs: a bar graph and a colored line graph. When the main 14 metrics we first presented, none of the structures had more than one metric encapsulated within the widget. Now widgets containing these complex forms with not only two but potentially more sub-graphs indicate a special correlation between them. Let us take a closer look at the figure 7 and determine why this graph was chosen to be present to the manager who is responsible for monitoring the dashboard. 


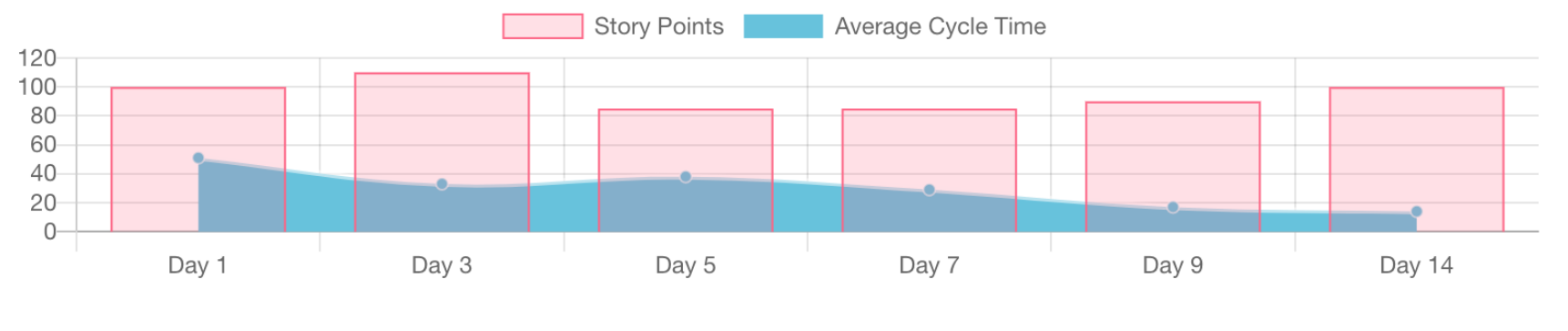

Fig. 7. Correlation between Story Points and Avg. Cycle Time

To start off, it is important to note that this graph spans the time period of exactly 14 days, the duration of one Sprint. Therefore, $\mathrm{X}$ axis represents the corresponding stage of the Sprint. Next, the Y axis is responsible for showing the amount of Story Points that a team has managed to obtain on the specific day. It also shows the Average Cycle Time of a task in the sprint (measured in minutes).

From the chart it is also easy to notice that the cycle time is fairly low, indicating that the tasks are well segmented and there are a lot of them, as the amount of story points is not low. However, the reason this graph is particularly interesting is that the Average Cycle Time graph is trending downwards. That means that it took more time for developers and designers to complete the tasks at the beginning of the Sprint rather than on the end of the Sprint.

There are numerous possible reasons for that, but the manager can be certain that his/her team did not try to hurry up at the Sprint end to finish most of the tasks because the number of Story Points is evenly distributed throughout the week. The manager might be mislead to thinking that that is the case only by looking at the Average Cycle Time. The combination of the two graphs frees him/her from that suspicion, and now he/she can focus on other, less critical reasons why that is the case.

Here are some of the possible reasons:

- the scope/size of the tasks is not uniformly distributed throughout the Sprint

- the team decided to first finish the complex tasks before moving on to easier ones

As one can see, neither of the two reasons are as critical as the team doing most of the work at the Sprint's end. Thereafter, the widget managed to effectively show that information without the manager wasting his/her precious time on tracking down the issue that did not exist in the first place.

This is an example of an effective combination of the two metrics that is far more useful when analyzed together rather than separately. It was determined to be important due to the fact that the trend was noticed in the Average Daily Cycle Time, and that such regularity did not cause any fluctuation in the number of Daily Story Points in the Sprint. Rather than looking at the raw data and realizing that his/her team is trying to do most of the work at the end of the Sprint, manager focused his/her attention to other problems and ways the workflow can be improved. The dashboard served as a helper tool to get the job done by enhancing workers' expertise and not getting in the way of already productive existing workflow pipelines.

\section{CONCLUSION}

We have shown our previous work in the field of Software Metrics and suggested a possible way of improving it, as well as to give rise to the importance of designing and developing compelling dashboards. We have previously decided on 14 key metrics for the software development process. Now, by continuous improvement and natural selection of the most relevant agents the dashboard should be able to achieve the dynamic equilibrium using non-linear dynamics. The dashboard would also be able to combine several metrics that have a higher chance of indicating a possible flaw in the workflow. That does not necessarily mean that it is going to achieve the optimal state right away, nor does it mean that just by applying the techniques presented here will we truly solve the problem of inadequate dashboards in general. But rather this all means that our dashboard will try to get there over time, by facilitating adaptation, collaboration and expertise while avoiding "chaos".

\section{Acknowledgments}

The work presented in this paper was supported by the grant of Russian Science Foundation №19-19-00623.

\section{REFERENCES}

[1] R. Brath and M. Peters. Dashboard design: Why design is important DM Direct, 85, 2004/10.

[2] I. D. Coman, A. Sillitti, and G. Succi. A case-study on using an automated in-process software engineering measurement and analysis system in an industrial environment. In Proceedings of the 31st International Conference on Software Engineering, ICSE '09, pages 8999, Washington, DC, USA, 2009. IEEE Computer Society.

[3] W. W. Eckerson. Performance Dashboards: Measuring, Monitoring, and Managing Your Business. Wiley, 2010. 
[4] S. Few. Information Dashboard Design: The Effective Visual Communication of Data. O'Reilly Media, 2006.

[5] D. Holten, R. Vliegen, and J. van Wijk. Visual realism for the visualization of software metrics. In 3rd IEEE International Workshop on Visualizing Software for Understanding and Analysis. IEEE, 2005.

[6] V. Ivanov, V. Pischulin, A. Rogers, G. Succi, J. Yi, and V. Zorin. Design and validation of precooked developer dashboards. In Proceedings of the 26th ACM Joint European Software Engineering Conference and Symposium on the Foundations of Software Engineering, ESEC/FSE 2018. ACM, 2018.

[7] V. Ivanov, A. Rogers, G. Succi, J. Yi, and V. Zorin. Precooked developer dashboards: What to show and how to use. In Proceedings of the 40th International Conference on Software Engineering: Companion Proceeedings, ICSE '18, pages 402-403, New York, NY, USA, 2018. ACM.

[8] A. Janes, A. Sillitti, and G. Succi. Effective dashboard design. Cutter IT Journal, 26:17-24, 012013.

[9] A. Janes and G. Succi. Lean Software Development in Action. Springer Berlin Heidelberg, 2014.

[10] Y. Lajoie, N. Teasdale, C. Bard, and M. Fleury. Attentional demands for static and dynamic equilibrium. Experimental brain research, 97(1):139-144, 1993.

[11] L. López, S. Martínez-Fernández, C. Gómez, M. Choraś, R. Kozik, L. Guzmán, A. M. Vollmer, X. Franch, and A. Jedlitschka. Q-rapids tool prototype: Supporting decision-makers in managing quality in rapid software development. In Lecture Notes in Business Information Processing, pages 200-208. Springer International Publishing, 2018.

[12] V. Pishulin. to clarify later. 2018.

[13] A. Sarikaya, M. Correll, L. Bartram, M. Tory, and D. Fisher. What do we talk about when we talk about dashboards? IEEE Transactions on Visualization and Computer Graphics, pages 1-1, 2018

[14] M. Staron, W. Meding, J. Hansson, C. Höglund, K. Niesel, and V. Bergmann. Dashboards for continuous monitoring of quality for software product under development. In Relating System Quality and Software Architecture, pages 209-229. Elsevier, 2014.

[15] J. M. T. Thompson, M. Thompson, and H. B. Stewart. Nonlinear dynamics and chaos. John Wiley \& Sons, 2002.

[16] A. Valiullin. Designing a dashboarding system for visualization of noninvasively collected metrics. 2018.

[17] O. M. Yigitbasioglu and O. Velcu. A review of dashboards in performance management: Implications for design and research. International Journal of Accounting Information Systems, 13(1):41-59, mar 2012.

[18] V. Zorin. to clarify later. 2017. 